\title{
Novel Optimization to Reduce Power Drainage in Mobile Devices for Multicarrier-based Communication
}

\author{
Shalini Prasad ${ }^{1}$, S. Balaji ${ }^{2}$ \\ ${ }^{1}$ Research Scholar, Jain Univeristy, Department of Electronics and Communication Engineering, City Engineering \\ College, Bengaluru, India \\ ${ }^{2}$ Centre for Incubation, Innovation, Research and Consultancy, Jyothi Institute of Technology, Tataguni, Off Karakapura \\ Road, Bengaluru, India
}

\begin{tabular}{ll}
\hline \hline \multirow{2}{*}{ Article Info } & ABSTRACT \\
\cline { 2 - 3 } Article history: & $\begin{array}{l}\text { With increasing adoption of multicarrier-based communications e.g. 3G and } \\
\text { 4G, the users are significantly benefited with impressive data rate but at the }\end{array}$ \\
Received Mar 18, 2017 & $\begin{array}{l}\text { cost of battery life of their mobile devices. We reviewed the existing } \\
\text { techniques to find an open research gap in this regard. This paper presents a } \\
\text { nevised Jun 4, 2017 }\end{array}$ \\
nccepted Aug 11, 2017 & $\begin{array}{l}\text { function to maintain higher level of equilibrium between maximized data } \\
\text { delivery and minimized transmit power. An analytical model considering } \\
\text { multiple radio antennae in the mobile device is presented with constraint } \\
\text { formulations of data quality and threshold power factor. The model outcome }\end{array}$ \\
3G & $\begin{array}{l}\text { is evaluated with respect to amount of power being conserved as } \\
\text { performance factor. The study was found to offer maximum energy }\end{array}$ \\
Battery lifetime & conservation and the framework also suits well with existing communication \\
Mobile device & system of mobile networks. \\
Multicarrier transmission &
\end{tabular}

Copyright $\odot 2017$ Institute of Advanced Engineering and Science. All rights reserved.

\section{Corresponding Author:}

Shalini Prasad

Research Scholar, Jain Univeristy,

Department of Electronics and Communication Engineering,

City Engineering College, Bengaluru, India

Email:- shaliniphdjain@gmail.com

\section{INTRODUCTION}

With the advancement of telecommunication standards, the users are now flooded with numerous services of wireless communication systems [1]. This has led to various forms of mobile applications that offer a good pervasive computing integrated with mobile networks [2]. A legacy $2 \mathrm{G}$ service uses a simple mechanism of transmitting the signal, which doesn't demand more power to be used by the mobile device. With evolution of $3 \mathrm{G}$ and $4 \mathrm{G}$ networks, there is a significant growth of multicarrier communication standards that potentially increases the data rates in mobile communication [3]. Usage of multicarrier-based communication standards offers higher data transmission that positively attracts users to use certain mobile applications that are highly inclined on performing parallel processing. Unfortunately, such forms of events go untracked by the users for which sufficient counter measures are difficult to be taken. Deployment of such forms of the network majorly uses OFDM (Orthogonal Frequency Division Multiplexing), which already suffers from PAPR (Peak-Average-to Power Ratio) [4] [5]. Therefore, usage of 3G or 4G network offers maximized rate of data transmission but at the cost of battery life of the mobile device. The existing mobile applications for power conservation are discretely programmed to identify certain specific threads that finally suppressed it [6]. Such a program doesn't help much in minimizing the battery consumption of a mobile device as it cannot identify the dynamic threads running over it. Hence, it is quite a challenging task to ensure the power conservation in the mobile devices. From the research viewpoint, it can be seen that there are very few computational models to identify the sources of power dissipation and to minimize it. Studies like [7] [8] 
[9] present some of the significant research work towards minimization of the power in the mobile device considering various types of networks e.g. sensor network, ad hoc network, etc. A wireless channel is also shrouded by various other types of problems e.g. scattering, interference, noise, fading etc. that adversely affect the signal quality at the receiver's end. Very less research has been found to be focused on minimization of power considering the network parameters. Another significant open research issue is the allocation of power while working on multicarrier-based communication system e.g. $3 \mathrm{G}$ or $4 \mathrm{G}$, where an optimal power is computed to be allocated on each subcarrier. This is a very important operation in all forms of futuristic telecommunication standards on which the modulation technique has to be focused. Usually, the modulation technique is very specific for type of the carrier used that finally results in more transmit power allocation from the mobile device. Hence, it can be finally stated that existing mobile networks offer data rate at the cost of mobile device's battery life. Control over transmit power of a wireless node is highly important design principle that can ensure uniform rate of energy dissipation. At the same time, energy will be required to be maintain a balance with traffic flow.The proposed system presents a novel framework that mainly targets to minimize such forms of transmit power of the mobile device when operating on multicarrier transmission in wireless communication systems. Section 1.1 discusses about the existing research work that has addressed the problem of power in the mobile device in the past by various researchers followed by a briefing of the problem identification in Section 1.2. Discussion of the contribution along with highlights of schematic architecture of the proposed system is made in Section 2 followed by research methodology in Section 3. The algorithm implementation is discussed in Section 4 and Section 5 discusses results being obtained. Section 6 concludes the paper by summarizing its outcome.

\subsection{Background}

This section discusses about the existing techniques of energy conservation in mobile devices. Our prior review work [10] discusses some of the standard models of energy management system. This section will further update our prior studies. Abolfazli et al. [11] discuss about various challenges in using cloud services over mobile devices. Although, the paper discussed about various augmentation techniques but it gave a fair insight about the restricted resources and capabilities of mobile devices. The most recent study of Ravichandran and Muralidharan [12] present a unique technique to control power efficiency by using dynamic frequency scaling approach. The technique was implemented on an Android device using ARM architecture. The study shows approximately 30\% reduction in power consumption. Pyles et al. [13] present a technique that is based on minimized delay and minimal power over the MAC layer. The contribution of the study is that the presented mechanism performs autonomous switching between two different standards of wireless networks, namely, Bluetooth and WLAN in order to conserve energy for the mobile devices. The study found approximately $25 \%$ of the energy saving. The study carried out by Bala and Garg [14] shows a technique for conserving the battery life for smart phones. The authors present a framework of learning that was used for monitoring the significant and unique behavior with respect to the energy drainage, frequencies of mobile applications being used, and different types of context using artificial neural network. However, the outcome is used for just checking the amount of energy being drained and not the solution for this. Tang et al. [15] propose a study where the problem of reduction in the tail energy is used by deploying machine learning techniques. The work has used an architecture using client and server module that applies training operation only on the server. The proposed system is designed over the actual prototype where the enhancement of the energy performance was obtained along with minimal overhead. In order to carry out this task, the authors have implemented both online and offline scheduling algorithms where the learning operation is carried out using an artificial neural network.

The study by Tilevich and Kwon [16] uses cloud offloading mechanism as well as various transformation techniques in cloud to obtain energy optimization. The offloading operation in cloud was carried out by disconnecting the cloud resources. Various forms of techniques used for cloud offloading operations are i) adaption of run time, ii) abstraction of programming, and iii) profiling and monitoring of runtime. Han et al. [17] propose a protocol called eDiscovery that is responsible for controlling the energy dissipation in opportunistic mobile networks. The protocol performs the alteration of the duration of the IEEE 802.11 standard for searching for the dynamic environment of the wireless networks. This approach was found to accomplish approximately $40 \%$ of the energy saving. The model implemented by the Kousai et al. [18] presents a technique of polar detection over the circuits of impedance detection. A loop of linearization is designed over the circuit for the purpose of reducing the communication overhead. A prototype is designed for carrying out the impedance detection using FPGA. Moety et al. [19] present a technique that addresses the problems of both power and delay in LTE (Long Term Evolution) networks. The system computes the transmit power by minimizing the cumulative power and communication delay. Study of power allocation towards advanced telecommunication standards like LTE was carried out by Nagaraj and Sarkar [20]. The technique performs reduction of power dissipation against the use of $4 \mathrm{G}$ network service $24 / 7$. The proposed 
system claims accomplishing $20 \%$ of reduction in the power as compared to the existing techniques. The study has also taken the case study of OFDMA-based mobile networks especially focusing on base station power reduction. $\mathrm{Wu}$ et al. [21] present a simple analytical framework that performs sharing of nodes to obtain energy efficiency. The authors have addressed the problem pertaining to resource sharing using a technique based on merging and splitting protocol.

Miao [22] presents a study that emphasizes on minimizing the energy conservation of multiple input and multiple output over uplink communication. A novel circuit management is designed and analysis is carried out in a hypothetical manner. Resource sharing is also one of the definitive alternatives of minimizing the power conservation over the mobile devices. The study by Mtibaa et al. [23] introduces an environment with collaborative nodes. The technique performs implementation of offloading schemes of cloud to enhance the battery longevity using an experimental approach. Qui et al. [24] carried out research using dynamic scheduling to maintain power efficiency in smartphones. The technique initially investigates the online jobs and its respective communication process using dynamic acyclic graphs. Yoo et al. [25] present a protocol for carrying out clustering operation between two different types of wireless networking standards. The technique also presents an explicit algorithm to perform election of cluster head, cater to the bandwidth requirements, etc. The study by Qian and Andresen [26] presents a technique that performs scheduling of various forms of tasks over the mobile devices especially with $4 \mathrm{G}$ networks. The study implements a framework using Jade that performs autonomous monitoring of various tasks along with application status by fine-tuning the dynamic strategies of the offloading of task. The mechanism was found to conserve approximately $35 \%$ of energy consumption.

Thus, it can be seen that there are various techniques found in the current literature focusing on minimizing the energy seepage from the mobile device. However, there is less standard and benchmarked research work which could be further used for future study. The next section discusses about the pitfalls in the reported literature and identifies the research gap and the potential problems for further research.

\subsection{The Problem}

On the basis of the review of the existing literature in the previous section, various shortcomings of the reported research contributions have been summarized. Some of the significant problems explored are as follows:

- Majority of the existing techniques are found to implement scheduling as one of the solution to counter the power problems on mobile devices. However, the scheduling was only studied with respect to a specific form of network e.g. Bluetooth or WLAN or data connection (i.e. $3 \mathrm{G}$ or $4 \mathrm{G})$. It is quite imperative that wireless standards do affect the battery life of a mobile device, but this problem was not found to be addressed.

- There are only few studies towards adoption of sophisticated optimization policies. Majority of the techniques do not address the trade-off between power minimization and maximizing throughput. Although, such problems are better mapped by non-linear optimization, very few researchers have ever considered such problems.

- Power seeps more when multimedia files are used on any mobile networks. Reported studies towards energy modeling are not found to consider profiling energy parameters based on such heavy multimedia contents over mobile devices.

- Lack of benchmarked studies and lack of regulatory standards on power usage is another significant research gap.

\section{PROPOSED STUDY}

This paper is a continuation of our prior mathematical modeling [27] which is focused on estimating the actual power dissipation from the mobile devices over existing telecommunication standards. This part of the study presents a framework that essentially performs minimization of the power dissipation in the mobile device. Figure 1 show the schematic architecture of the proposed study which mainly consists of system model and communication model. The system model consists of a number of radio antennas in mobile devices, instantaneous power, tolerance factor, and channel parameters. The system considers a signal that acts as an input to transmitter which is further subjected to modulation. Multicarrier transmission is considered to accommodate all forms of modulation. The encoded and modulated signal is now subjected to channel with noise and interference, which is finally subjected to an optimization algorithm. By mapping to the problem of non-linear optimization, a novel objective function is developed to properly balance between data rate and transmit power. The algorithm designed for this purpose also formulates constraints pertaining to threshold power and data quality, which is mainly considered as SNR (Signal-to-Noise Ratio) and BER 
(Bit-Error-Rate). The entire work is carried out during the modulation operation itself as this mechanism has to be incorporated within the hardware of the mobile device. Hardware profiling potentially assists the algorithm to make an efficient data aggregation for usage as well as network behavior too. Another interesting part of the study is that the proposed system has faster response time and suits well for lower bandwidth availability too. The next section elaborates about the adopted research methodology followed by algorithm implementation.

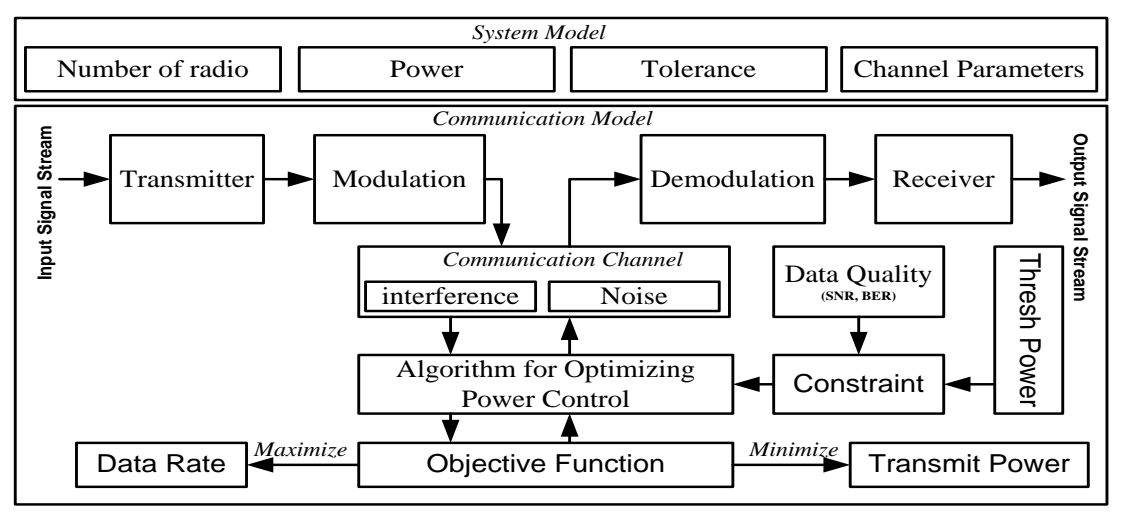

Figure.1. Schematic Architecture of the Proposed System

\section{RESEARCH METHODOLOGY}

The design and development of the proposed system is carried out using analytical design methodology. In general, there are 5 types of radio antenna used in smartphones: (i) Wi-Fi antenna, (ii) FM Radio antenna, (iii) Main antenna, (iv) Diversity antenna, and (v) GPS antenna as shown in Figure 2. These antennae are meant to serve different purposes. For example, Wi-Fi antenna connects the device with an access point but GPS antenna connects the device with GPS. Hence, they can be grouped as cellular RFbased antenna and Mobile connectivity RF-based antenna. The design of the proposed system is done with one of the challenging assumption that all antennae are functioning at the same time. These assumptions will lead to a scenario of maximized power drainage, which will be ideal to prove the robustness and sustainability of proposed power control-based optimization technique to hold right. The proposed system considers design of a channel matrix $\mathrm{H}$, where $y=H . s+n$ ( $y$ being user stream data at receiver and $s$ being that of transmitter).

As known from the concepts of MIMO[28] at high SNR, the capacity of the i.i.d. (independent and identically distributed) Rayleigh fast fading channel scales like $\mathrm{n}_{\min } \log$ SNR bits/s/Hz, where $\mathrm{n}_{\min }$ is the minimum of the number of transmit antennae $\mathrm{n}_{\mathrm{t}}$ and the number of receive antennae $n_{r}$. This is a degree-offreedom gain. At low SNR, the capacity is approximately $\mathrm{n}_{\mathrm{r}} \cdot \mathrm{SNR} \cdot \log _{2} \mathrm{e}$ bits/s/Hz [28]. This is a receive beam-forming power gain. At all SNR, the capacity scales linearly with $\mathrm{n}_{\min }$. This is due to the combination of the power gain and the degree-of-freedom gain.

$$
\mathrm{y}=\mathrm{Hs}+\mathrm{n}
$$

Let the transmitted vector $\mathrm{s}$ be a random vector to be very general and $\mathrm{n}$ is normalized noise. Let the total transmitted power available per symbol period be $\mathrm{P}$. The instantaneous channel capacity can be given as

$$
\mathrm{C}=\log _{2}\left(\mathrm{I}_{\mathrm{M}}+\mathrm{HQH}^{\mathrm{H}}\right) \mathrm{b} / \mathrm{s} / \mathrm{Hz}
$$

We consider a frequency flat MIMO channel to calculate its capacity. Where $I_{M}$ is a unit matrix $N_{R}$ $X N_{R}, H$ is the channel matrix $N_{R} X N_{T}$ and $Q=E\left\{s s^{H}\right\}$ the covariance matrix of transmitted signal $s$ satisfying the transmit power constraint $\operatorname{Tr}\left(\mathrm{ss}^{\mathrm{H}}\right)=\mathrm{N}_{\mathrm{T}}$. Consider specific case when we have users transmitting at equal power over the channel and the users are uncorrelated (no feedback available). Then, the channel capacity is

$$
\mathrm{C}_{\mathrm{EP}}=\log _{2}\left[\mathrm{I}_{\mathrm{M}}+\left(\mathrm{P} / \mathrm{M}_{\mathrm{T}}\right) \mathrm{HHH}\right] \mathrm{b} / \mathrm{s} / \mathrm{Hz}
$$


The proposed system uses a mechanism of antenna selection based on a unique objective function, which can be mathematically represented as,

$$
O_{\text {func }}=\left[\operatorname{Max} \sum_{k=1}^{\eta} \operatorname{data}, \operatorname{Min} \sum_{k=1}^{\eta} \rho\right]
$$

A closer look into the above objective function will show that proposed FoPC (Framework for Power Control) maintains a better fairness by balancing both data delivery as well as power required for transmitting data $(\rho)$. Considering the challenges of wireless networks, the study considers that above stated objective function only for specific rate of error $\left(\mathrm{e}_{\mathrm{i}}<\mathrm{e}_{\mathrm{TH}}\right)$. The proposed system thereby performs optimization without using any forms of additional resources to control the power dissipation of the mobile devices. In the mathematical model, a new variable weight is defined that is responsible for adjusting the transmission rate on multiple radio antennas thereby controlling the power dissipation to a large extent. The computational complexity of non-linearity is reduced using Lagrangian multiplier while a slack variable is used for transforming non-linear to linear forms for minimizing the complexities.

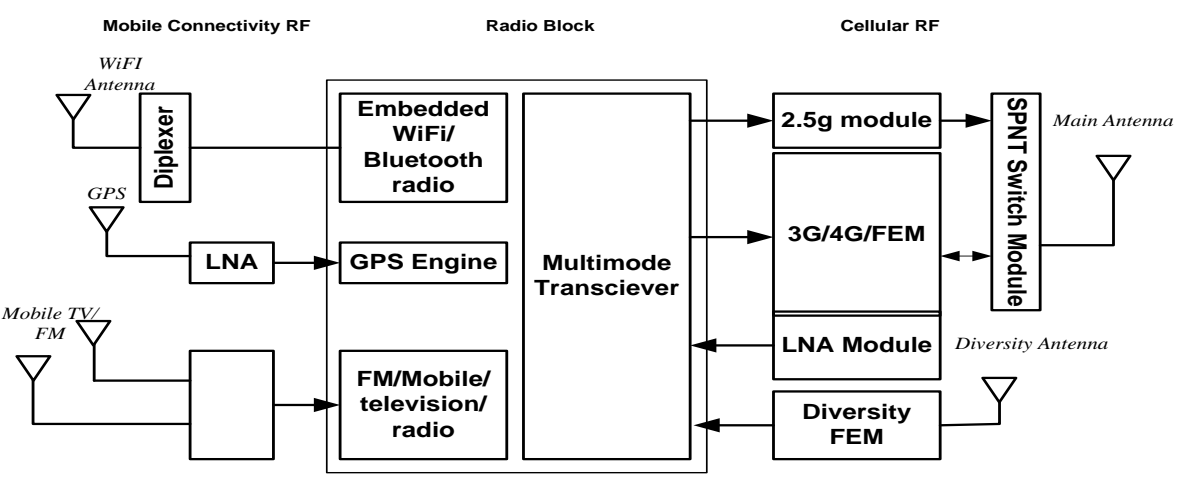

Figure.2. General Circuit Diagram of Smartphone with Radio Antenna

\section{ALGORITHM IMPLEMENTATION}

The prime purpose of the proposed algorithm is to ensure that there is a good balance between the power conservation, data quality, and communication performance. Mobile devices have multiple radio antennas that work simultaneously. Each channel selected by the radio interface has a specific amount of power allocation and hence more the communication or data transmission more is the power dissipation. Thus, the primary aim of this algorithm is to address the tradeoff between data delivery maximization (using multiple radios) and minimization of power that will be used for transmitting data from the mobile device. The steps of the algorithm are as shown:

Algorithm for Optimizing Power Control

Input: $\eta$ (Number of radio), $\alpha$ (Weight of channel), $e_{T H}$ (threshold error), $\rho$ (Power), $\tau$ (Tolerance), $\varphi$ (Data Quality), $d$ (data), $T_{x}$ (Transmitter antennae), $\lambda$ (Lagrangian Multiplier), $\delta$ (Slack variable), $H_{i}$ (Channel matrix), $n_{v}$ (noise variance), $C_{v r}$ (Channel-variance ratio), $\varepsilon_{l} / \varepsilon_{2}$ (Channel Parameters). Output: $o_{\text {pow }}$ Optimized power).

\section{Start}

1. Define $\eta, \alpha, \rho, \tau, \varphi, d$.

2. Init $\mathrm{T}_{\mathrm{x}}$,

3. $T_{x} \stackrel{\text { def }}{\longrightarrow}\left(\lambda, \delta, e_{T H}\right), \forall H_{i}=\eta$

4. For $\mathrm{i}=1: \varphi$

5. $n_{v}=\sqrt{10^{-\frac{\phi}{10}}}$

6. $\quad C_{v r}=\left|H_{i}\right|^{\frac{2}{n_{v}^{2}}}$

7. $\quad t_{1} \Rightarrow \sum_{j=1}^{\eta}\left[\lambda \cdot \varepsilon_{1} \cdot \exp \left(\frac{\varepsilon_{2} \cdot C_{v r} \cdot \rho}{2^{d}-1}\right)-e_{T H}+\delta^{2}\right]$

8. $\quad t_{2} \Rightarrow \sum_{j=1}^{\eta} \alpha . \rho$ 
9.

$$
t_{3} \Rightarrow \sum_{j=1}^{\eta}(1-\alpha) \cdot d
$$

10.

$$
o_{\text {pow }} \rightarrow \frac{t}{\max (t)}, t=t_{1}+t_{2}+t_{3}
$$

11. End

End

The proposed algorithm takes the input of $\eta$ (number of radios), $\alpha$ (weight of channel), $e_{T H}$ (threshold error), $\rho$ (power), $\tau$ (tolerance), $\varphi$ (data quality), $d$ (data), $T_{x}$ (transmitter antennae), $\lambda$ (Lagrangian multiplier), $\delta$ (slack variable), $H_{i}$ (channel matrix), $n_{v}$ (noise variance), $C_{v r}$ (channel-variance ratio), $\varepsilon_{l} / \varepsilon_{2}$ (channel parameters), etc. and after processing

Results in $o_{\text {pow }}$ (optimized power). The algorithm works by initially defining the variables (e.g. $\eta, \alpha$, $\rho, \tau, \varphi$, d.) in Line-1, whose values depend on the type of application, type of network and services to be used by the mobile devices. The algorithm basically runs over mobile devices and is mainly focused on minimizing transmit power. As power required to transmit is slightly higher than power required to receive, the present algorithm emphasizes that the algorithm execution to take place at the transmitting mobile device i.e. $\mathrm{T}_{\mathrm{x}}$ (Line-2). The transmitting node will also need to define certain critical variables e.g. $\lambda$ (Lagrangian multiplier), $\delta$ (slack variable), and $\mathrm{e}_{\mathrm{TH}}$ (cut-off error rate) (Line-3). The reason behind this is: addressing the trade-off between throughput maximizing and minimization of transmit power is basically a non-linear optimization problem considering various factors such as channel noise, signal power, data rate, capacity of the channel and channel attenuation. Hence our computation becomes complex considering all the parameters. Therefore, the algorithm uses Lagrangian multiplier to minimize the computational complexity of the algorithm by exploring the local maxima as well as local minima with the data quality constraint. The slack variable is used for transformation of non-linear to linear constraint. The prime motive behind Line-3 is to make the algorithm feasible to implement in a cost-effective manner by considering ideal state of channel $\mathrm{H}_{\mathrm{i}}$ which is equivalent to number of radio antennae itself. The next step of algorithm implementation begins with a loop that represents the constraints of data quality (Line-4 - Line-11). For this reason, the algorithm computes the noise variance $n_{v}$ in Line- 5 followed by computation of channel to noise variance $C_{v r}$ in Line- 6 . Then, the algorithm implements its mathematical model shown in Line-7, Line-8, and Line-9, which is based on constraint of error rate, power, and data to be transmitted. Finally, it computes the optimized power in Line-10 at which the data can be transmitted, hence ensures an effective power control in mobile device.

Table.1. Symbol and Notation used

\begin{tabular}{ll}
\hline$\eta$ & Number of radio \\
$\alpha$ & Weight of Channel \\
$\mathrm{e}_{\mathrm{TH}}$ & threshold error \\
$\rho$ & Power \\
$\tau$ & Tolerance \\
$\varphi$ & Data Quality \\
$\mathrm{d}$ & Data \\
$\mathrm{T}_{\mathrm{x}}$ & Transmitter antennae \\
$\lambda$ & Lagrangian Multiplier \\
$\delta$ & Slack variable \\
$\mathrm{H}_{\mathrm{i}}$ & Channel matrix \\
$\mathrm{n}_{\mathrm{v}}$ & noise variance \\
$\mathrm{C}_{\mathrm{vr}}$ & Channel-variance ratio \\
$\varepsilon_{1} / \varepsilon_{2}$ & Channel Parameters \\
$\mathrm{O}_{\mathrm{pow}}$ & Optimized power \\
\hline
\end{tabular}

\section{RESULTS AND DISCUSSION}

This section discusses about the results being obtained from the implementation of the proposed system. The entire implementation of the proposed system is carried out on a 32-bit Windows machine with Core i3 processor and 4GB RAM. The results presented here consider i) No. of radio antennae used $=8$, ii) Weight adopted $=0.5$, iii) Error Threshold $=0.0001$, iv) Data Quality $=-30 \mathrm{~dB}$ to $+30 \mathrm{~dB}$, v) Tolerance $=1 \mathrm{e}$ 09 , vi) Power allocated to each radio antenna $=1$ Joule, and vii) data allocated to each radio $=10$ Megabytes. The implementation is carried out in Matlab where a transmitter, receiver, and a wireless channel with multiple radio antennae are designed. The implementation also uses a fading and noisy wireless channel for propagation in order to check the effectiveness of the system. A series of standard signals (in the form of an 
image) is transmitted from transmitter to check the performance. Figure 3 shows an image taken as input signal for experiment that is transmitted by the wireless channel corrupted by additive noise.

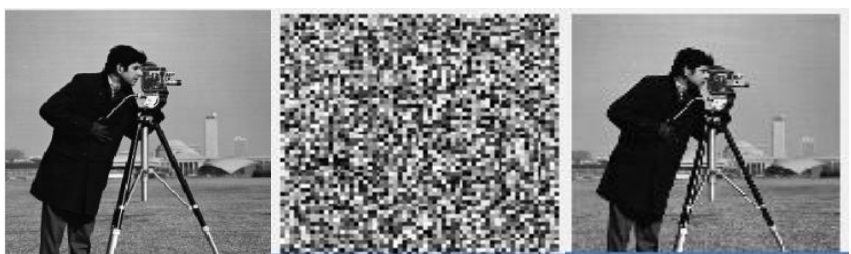

Figure.3. Input image, Energy Optimization and Received Image

For both consistent and irregular traffic, we investigated multiple situations for evaluating its dependencies on the channel model. The system also utilized a static arrangement with all receiving dynamic antennae as the standard technique to evaluate the antennae management systems of the mobile device. The power per bit of transmitting node is measured which executes antennae system management and transmits 1000 data segments to receiving node. For both estimations, we used four diverse data rates of $0(\sim 0.119), 100$, 200, and $300 \mathrm{Mbps}$

\subsection{Analysis for Continuous Traffic}

This assessment was conducted in order to understand the pattern of power dissipation when subjected to streaming of contents. Figure 4(a) and Figure 4(b) highlight the performance with respect to transmitter and receiver, respectively. For continuous traffic, the transmitter (Figure 4(a)) shows that energy consumption has been reduced to higher extent with increased rate ( $\mathrm{r}=0-300 \mathrm{Mbps})$. The receiver too shows similar performance (Figure 4(b). Initially, the energy per bit accomplished by receiving antennae for the mobile device is smaller than that of the static design. Secondly, with the increase in rate of data, there is a drop of energy per bit by the receiving nodes. Third, under a moderately low rate of data (e.g. under 200 Mbps) antennae system management turns out to be more successful. This is due to the fact that channels with high data rate require more receiving antenna on device which in turn leads to less change in the capacity yet causes extra power utilization. Another significant reason for such uniformity in transmitter and receiver performance is that continuous traffic is quite predictable and hence the algorithm can easily apply equivalent channel capacity with deterministic priority of the sub-channels (in case of increasing rate of flow) to cater to the increasing traffic demands. Hence, with increasing number of antennae, the proposed system can successfully reduce the energy per bit. The robustness of energy efficiency is proven as with increasing of minimum rate value, the capability of rate reduction still increases. Therefore, there is no negative impact on the data quality in streaming communication channel.

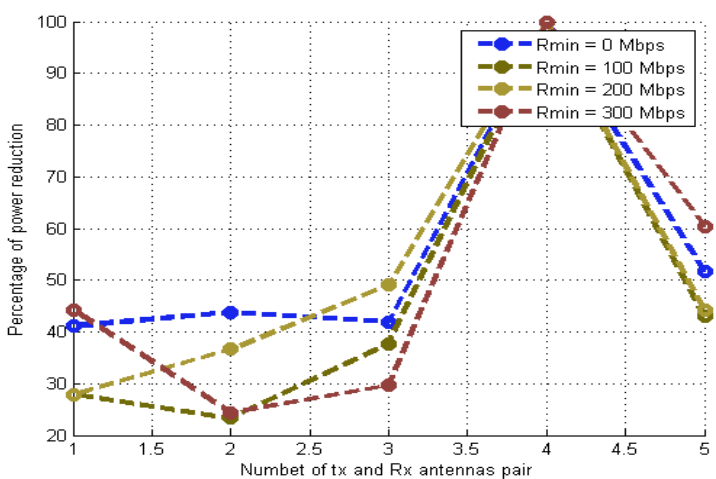

Figure.4.(a) Performance of Power Reduction in Transmitter

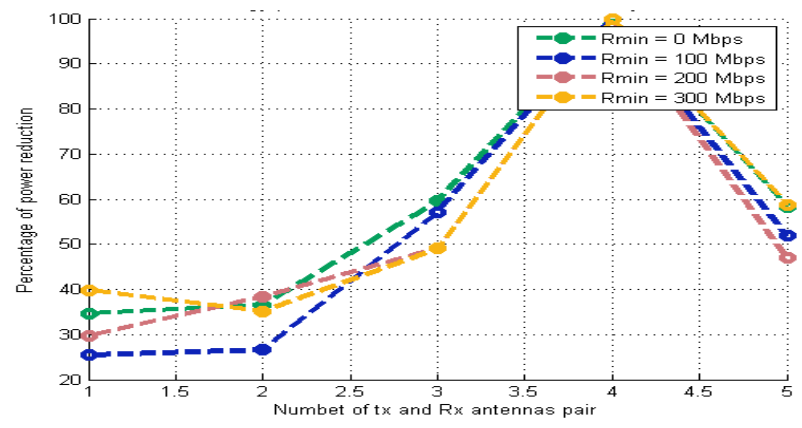

Figure.4.(b) Performance of Power Reduction in Receiver

Figure.4. Energy per Bit Reduction 


\subsection{Analysis for an Intermittent Traffic}

Basically, intermittent traffic is a result of connectivity outage because of large number of online users whose services are quite difficult to be scheduled dynamically. Such cases usually result in drop of data packet at the end. However, the matter of concern is that a massive and highly dynamic task requests from the user are sometimes difficult to handle and in order to retain optimum connectivity the RRC (Radio Resource Control) allocates more power on channel resulting in more power dissipation. Hence, an intermittent traffic is selected for the assessment. A similar assessment scenario of transmitter node and both transmitter-receiver node is considered intermittent traffic will mean uncertainty due to non-uniform traffic flow towards the nodes. This will cause uneven distribution of energy dissipation of the node. This problem can be only solved by objective function where it will need to keep lower amount of transmittance energy with higher traffic flow from each node. Hence, transmitter and receiver performance are not same in this case. The transmitter node will need to allocate precise resource (energy, channel capacity), where Figure5 (a) shows that transmitter doesn't seem to normalize the energy reduction to a significant extent. Although, it is successful to minimize the power progressively for higher rate. This phenomenon also uses certain extent of transmittance energy (on the transmitter side) will have positive effect on receiver (Figure 5(b)). The results depicted in Figure 5 show that proposed system provides higher sustainability of battery longevity even on intermittent traffic. At the same time, it does not have any negative impact on the data rate from 0-300 Mbps exhibiting a robust antenna management and energy conservation.

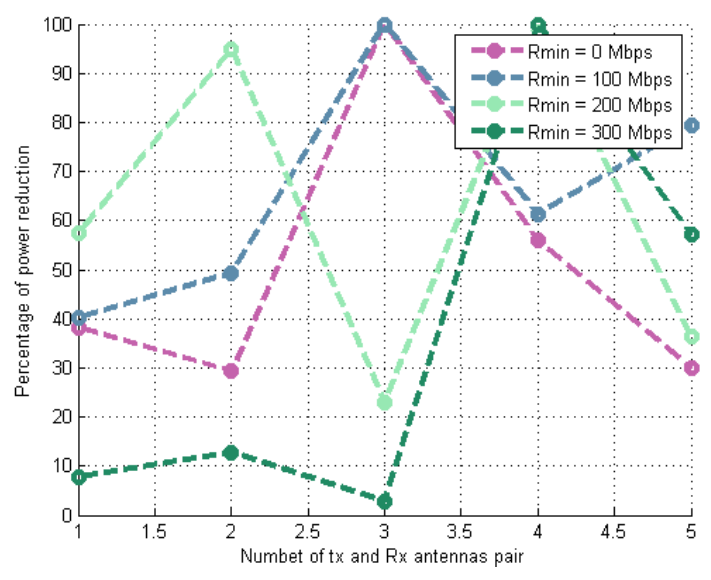

Figure.5(a). Performance of Power Reduction in Transmitter

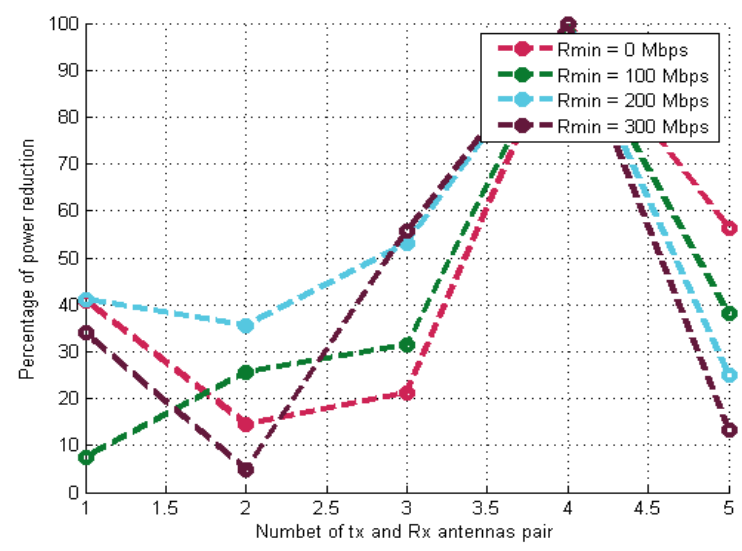

Figure.5(b). Performance of Power Reduction in Receiver Figure.5. Energy per bit reduction for one end Intermittent

Figure 4 shows that proposed system offer highly better performance in continuous traffic situation whereas in intermittent traffic situation, it offers better energy conservation to the receiver node .The 
outcome of the proposed system was also compared with the work carried out by Zhang et al. [29]. A framework by the name E-MiLi by Zhang et al focuses on curtailing the idle listening power of the node in wireless networks using sleep-scheduling based optimization. E-MiLi also uses a clocking mechanism that significantly assists in MAC-layer scheduling. The framework showed approximately 40-90\% of reduction in energy consumption for real-time users. In order to perform comparative analysis, minor amendments were carried out in switching the variables used in E-MiLi to fit into proposed system (FoPC). The comparative results of our framework with that of E-MiLi is shown in Figure 6, where it can be seen that proposed system offers better resiliency against power drainage in comparison to that of existing system with increasing data quality (in dB). E-MiLi does not perform any form of optimization in case of multiple traffic situations but the proposed system is able to identify the type of traffic and perform resource allocation based on this. It can also maintain a ceiling for energy consumption and leverage traffic flow, which cannot be seen in E-MiLi. A closer look shows that E-MiLi exhibits data quality right from $10 \mathrm{~dB}$ whereas the proposed FoPC offers much better data quality from $25 \mathrm{~dB}$. Moreover, transmit power increases gradually and retains nearly constant for E-MiLi but at the cost of poor data quality. Thus, E-MiLi is not able to address the tradeoff between data quality and power conservation for mobile devices.

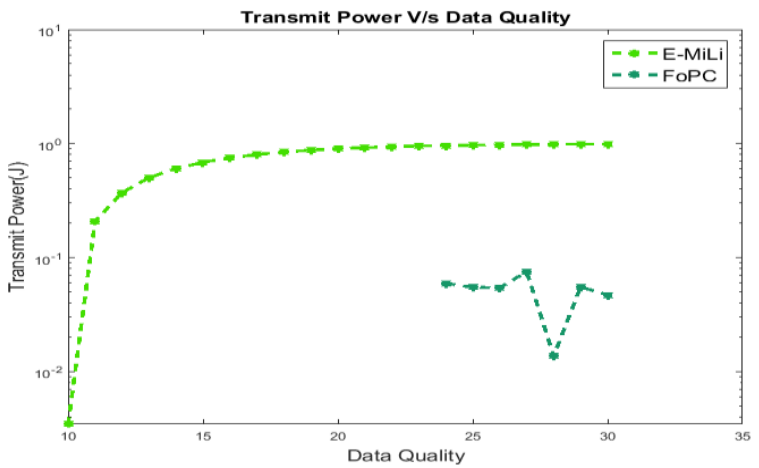

Figure.6. Comparative Performance Analysis of Transmit Power

The prime reason behind the better performance of proposed FoPC is that its algorithm introduces better optimization in its objective function that significantly balances maximization of throughput and minimization of power conservation. Therefore, the proposed system of FoPC can successfully address the power dissipation problems of the mobile devices operating under a challenging wireless network condition.

\section{CONCLUSION}

The hardware circuitry of a mobile device consists of sensing unit, communication unit, processing unit, and power supply unit. Interestingly, all these units have different power requirements from one external battery. Apart from various physical tasks in a mobile device, basic reason of power drainage in smartphones is also the type of the networks chosen by the user. As majority of the users primarily give importance to speed of network and are often found to migrate to $3 \mathrm{G}$ and $4 \mathrm{G}$ services. Such forms of network services use multicarrier-based communication system where different forms of transmit power allocation is made on each sub-carrier. After reviewing the existing system, it was found that no reported literature has focused on this problem. Hence, although the user enjoys higher data speed, their battery drains too fast. Therefore, our scheme presented in this paper acts as a barrier against such power seepage especially on multicarrier networks. We have presented a model where constraints of data quality and cut-off power are considered and a non-linear optimization problem is formulated by the proposed analytical model. Our architecture is highly scalable and responds fast within 0.2675 to 0.5344 seconds which complies quite well with existing multicarrier based communication standards. We have Implemented the model on Matlab, the outcomes were tested over various scenarios of communication channel (continuous and intermittent traffic inflicted with standard channel condition e.g. interference and fading) to find that it excels better communication performance as compared to existing techniques.

\section{REFERENCES}


[1] I. Robertson, N. Somjit, M. Chongcheawchamnan, Microwave and Millimetre-Wave Design for Wireless Communications, John Wiley \& Sons, 2016

[2] C. Dobre, F. Xhafa, Pervasive Computing: Next Generation Platforms for Intelligent Data Collection, Morgan Kaufmann, 2016

[3] M. Barreiros, P. Lundqvist, QOS-Enabled Networks: Tools and Foundations, John Wiley \& Sons, 2016

[4] P. Varahram, S. Mohammady, B. Mohd Ali, N. Sulaiman, Power Efficiency in Broadband Wireless Communications, CRC Press, 2014

[5] M. Rupp, S. Schwarz, M. Taranetz, The Vienna LTE-Advanced Simulators: Up and Downlink, Link and System Level Simulation, Springer, 2016

[6] "Battery - DU Battery Saver",https://play.google.com/store/apps/details?id=com.dianxinos.dxbs\&hl=en, Retrived, 11th May, 2017

[7] V. P. Singh, K. Kumar, "Literature Survey on Power Control Algorithms for Mobile Ad-hoc Network", Springer Journal, 2010

[8] J. Wu, Y. Zhang, M. Zukerman, "Energy-Efficient Base-Stations Sleep-Mode Techniques in Green Cellular Networks: A Survey", IEEE Communication Surveys \& Tutorials, vol. 17, no. 2, second quarter 2015

[9] M. N. Jamblia, H. Lenandoa, K. Zena, S. M. Suhailib, A. Tullyc, "The effects of transmission power control in mobile ad-hoc sensor networks", ScienceDirect-International Symposium on Robotics and Intelligent Sensors, vol.41, pp.1244, 1252 .

[10] S. Prasad, R. Sharma, and S.Balaji, "Brief Overview of Smartphone Power Consumption in Various Applications", Proceedings of International Conference on Multimedia Processing, Communication, 2013

[11] S. Abolfazli, Z. Sanaei, E. Ahmed, "Cloud-Based Augmentation for Mobile Devices: Motivation, Taxonomies, and Open Challenges”, IEEE Communications Surveys \& Tutorials, vol. 16, no. 1, first quarter 2014

[12] R. Ravichandran, V. Muralidharan, "A Dynamic Algorithm to Improve Power Efficiency in Smartphones", IEEEInternational Conference on Computer Communication and Informatics, 2016

[13] A. Pyles, D. T. Nguyen, X. Qi, and G. Zhou, "Bluesaver: A Multi PHY Approach to Smartphone Energy Savings", IEEE Transactions On Wireless Communications, 2015

[14] R. Bala, A. Garg, "Battery Power Saving Profile with Learning Engine in Android Phones", International Journal of Computer Applications, Vol.69, No.13, May 2013

[15] Z. Tang, S. Guo, P. Li, "Energy-Efficient Transmission Scheduling in Mobile Phones Using Machine Learning and Participatory Sensing", IEEE Transactions On Vehicular Technology, vol. 64, no. 7, july 2015

[16] E. Tilevich and Y-W Kwon, "Cloud-Based Execution to Improve Mobile Application Energy Efficiency", IEEE Computer Society, 2014

[17] B. Han, J. Li, and A. Srinivasan, "On the Energy Efficiency of Device Discovery in Mobile Opportunistic Networks: A Systematic Approach", IEEE Transactions On Mobile Computing, 2014

[18] S. Kousai, K. Onizuka, J. Wadatsumi, "Polar Antenna Impedance Detection and Tuning for Efficiency Improvement in a 3G/4G CMOS Power Amplifier", IEEE Journal Of Solid-State Circuits, vol. 49, no. 12, December 2014

[19] F. Moety, S. Lahoud, B. Cousin, K. Khawam, "Joint Power-Delay Minimization in 4G Wireless Networks", IEEEIFIP Wireless Days, 2014

[20] S. Nagaraj and M. Sarkar, "A Novel Power Allocation Technique for 4G Cellular Networks", IEEE International Conference on Computing, Networking, Communications, Wireless Communications Symposium, 2014

[21] D. Wu, J. Wang, R. Q. Hu, "Energy-Efficient Resource Sharing for Mobile Device-to-Device Multimedia Communications", IEEE Transactions On Vehicular Technology, vol. 63, no. 5, June 2014

[22] G. Miao, "Energy-Efficient Uplink Multi-User MIMO”, IEEE Transactions On Wireless Communications, vol. 12, no. 5, May 2013

[23] A. Mtibaa, A. Fahim, K. A. Harras, M. H. Ammar, "Towards Resource Sharing in Mobile Device Clouds: Power Balancing Across Mobile Devices", Proceedings of second ACM SIGCOMM workshop on Mobile cloud computing, vol.43, Iss.4, pp.51-56, 2013

[24] M. Qiu, Z. Chen, L. T. Yang, X. Qin, B. Wang, "Towards Power-Efficient Smartphones by Energy-Aware Dynamic Task Scheduling”, IEEE International Conference on High Performance Computing and Communications, 2014

[25] J-W Yoo, K. H. Park, "A Cooperative Clustering Protocol for Energy Saving of Mobile Devices with WLAN and Bluetooth Interfaces”, IEEE Transactions On Mobile Computing, vol. 10, no. 5, April 2011

[26] H. Qian, D. Andresen, “An Energy-saving Task Scheduler for Mobile Devices", IEEE International Conference on Computer and Information Science, 2015

[27] S. Prasad, S. Balaji, "Real-Time Energy Dissipation Model for Mobile Devices", Springer-Emerging Research in Computing, Information, Communication and Applications, pp.281-288, 2015

[28] P. Viswanath, D. Tse, "Fundamentals of Wireless Communication ” ISBN: 9781107386051 , Publisher: Cambridge University Press, 2005

[29] X. Zhang and K. G. Shin, "E-MiLi: Energy-Minimizing Idle Listening in Wireless Networks", IEEE Transactions on Mobile Computing, vol.11, Iss.9, pp.1441-1454, 2012 